\title{
Discapacidad, Inclusión y Educación Superior en Ecuador: El Caso de la Universidad Católica de Santiago de Guayaquil
}

\author{
Disability, Inclusiveness and Higher Education in Ecuador: The \\ Case of Universidad Católica de Santiago de Guayaquil
}

\author{
Juan Carlos Ocampo * \\ Universidad Católica de Santiago de Guayaquil, Ecuador
}

\begin{abstract}
Durante siglos, la universidad fue un espacio altamente excluyente y pese a los avances, aún hay vestigios remanentes de la época de discriminación, los cuales dificultan el ingreso, permanencia y egreso de ciertos colectivos históricamente marginados, como las personas con discapacidad. Este artículo presenta el caso de la Universidad Católica Santiago de Guayaquil en torno a la inclusión y discapacidad. Se plantea como objetivo i) describir el interés de la comunidad académica de la institución en torno a la discapacidad y ii) analizar las características del estudiantado con discapacidad de las cohortes desde el año 2013 hasta el 2017. Para ello se realizó un estudio descriptivo de la producción científica de los miembros de la institución y un análisis de los registros de estudiantes con discapacidad. Los resultados sugieren que hay un interés creciente en el tema por parte de los graduandos de la institución. También indican que no existen diferencias considerables en el ingreso de estudiantes con discapacidad según su género o provincia de origen, pero sí por grado y tipo de discapacidad. Además, son infrarrepresentados en cierto tipo de carreras. El ingreso de estudiantes con discapacidad a esta universidad ha ido en aumento desde el 2013, pero hasta la fecha no ha alcanzado el 1\% de la población estudiantil. Por último, se exhorta a investigadores, docentes e instituciones a emprender iniciativas de inclusión educativa en pos de una sociedad ecuatoriana más íntegra, solidaria y justa.
\end{abstract}

Descriptores: Discriminación; Discapacidad; Enseñanza superior; Universidad; Ecuador.

For centuries, universities were a highly exclusive space and despite the advances, there are vestiges remaining from the era of discrimination, which makes it difficult for historically marginalized groups to be admitted, stay and egress, such as people with disabilities. This article presents the case of the Universidad Católica de Santiago de Guayaquil on inclusivity and disability. The objective is to i) describe the interest of the academic community of the institution regarding disability and ii) analyze the characteristics of students with disabilities in the cohorts from 2013 to 2017. For this, I carried out a descriptive study on the scientific production of the members of this institution and an analysis of the records of students with disabilities. The results suggest that there is a growing interest in the subject by the graduating students of the institution. They also indicate that there are no significant differences in the admission of students with disabilities according to their gender or province of origin, but there is one by grade and type of disability. In addition, they are underrepresented in certain types of careers. The admission of students with disabilities to this university has been increasing since 2013, but it has yet to reach the $1 \%$ of the student population. Finally, researchers, teachers and institutions are urged to undertake educational inclusion initiatives in pursuit of a more integral, solidary and just Ecuadorian society.

Keywords: Discrimination; Disabilities; Higher education; University; Ecuador.

*Contacto: juan.ocampoo1@cu.ucsg.edu.ec

ISSN: 0718-7378

www.rinace.net/rlei/
Recibido: $30 / 05 / 2018$

$1^{\text {a }}$ Evaluación: 15/07/2018

Aceptado: 20/08/2017 


\section{Introducción}

El término inclusión ha permeado en todos los niveles de la sociedad, desde el discurso coloquial hasta la actuación política; pero, aunque esté presente en nuestro hablar cotidiano, su práctica es significativamente distinta. La inclusión está ausente en su mayor parte y todavía hay mucho camino por recorrer en vías de una sociedad más igualitaria. Esto es especialmente cierto en Latinoamérica donde, a pesar de robustos marcos legales que promulgan la inclusión, no se ha logrado una transformación profunda. Uno de los ámbitos con el menor progreso es el de la educación superior. Esto es particularmente preocupante porque, en la sociedad contemporánea, la universidad funge como preámbulo de la inserción laboral y requisito para la plena incorporación en el espacio social. Mientras existan limitaciones en el acceso y permanencia de las personas con discapacidad en la universidad, no habrá una completa integración en la vida social.

En el presente artículo se abordará la temática de la inclusión de personas con discapacidad en la educación superior en el caso específico de una universidad ecuatoriana. Para ello, en primer lugar, se expone una recopilación de estadísticas con el objetivo de establecer la prevalencia de la discapacidad a nivel nacional e internacional. A continuación, se describe el desarrollo histórico de la educación respecto a la discapacidad, a fin de introducir el concepto de inclusión. Después, se caracteriza la situación de las personas con discapacidad en la educación superior ecuatoriana, considerando el régimen jurídico, las estadísticas e investigaciones al respecto. Con base en lo expuesto, los objetivos del presente artículo son i) describir el conjunto de investigaciones realizadas por la comunidad académica de la universidad estudiada en torno a la discapacidad y ii) analizar las características del estudiantado con discapacidad desde el 2013 al 2017.

\subsection{Estadísticas nacionales e internacionales sobre la discapacidad}

El último informe de la OMS (2011) sobre la discapacidad destacó que alrededor del 15\% de la población mundial sufre de alguna forma de discapacidad, siendo los países de ingresos bajos aquellos de mayor prevalencia; dichas personas tienen una menor probabilidad de ser escolarizadas; y de la misma manera, tienen una menor probabilidad de ser empleadas. Pese a que las estadísticas varían considerablemente entre países, dichas consideraciones se mantienen. La discapacidad es un fenómeno prevalente en todas las regiones del mundo que afecta directa e indirectamente a un porcentaje significativo de la población.

En Estados Unidos, el 12,6\% de la población tiene una discapacidad y aunque la mitad de ellos se encuentren en edad productiva, sólo el 34,9\% está trabajando con una remuneración promedio un tercio menor que la de sus contrapartes sin discapacidad (Kraus, 2017). En el Reino Unido, aproximadamente el 19\% de la población general y el $16 \%$ de la población en edad productiva tiene una discapacidad, pero de estos últimos, el $44,3 \%$ es económicamente inactivo, cifra cuatro veces mayor que la de personas sin discapacidad (Smith, 2016). En España, el porcentaje estimado de personas con discapacidad varía de $9 \%$ a $19,7 \%$ según la encuesta y la tasa de empleo de las personas con discapacidad en edad activa (16 a 64 años) es de sólo el 23,4\% (Jiménez y Huete, 2016). Actualmente, la información sobre discapacidad en muchos países es deficiente y en otros casos, pese a las mejoras, sigue siendo limitada.

En el 2001 se incluyó la primera pregunta sobre discapacidad en el censo ecuatoriano (Instituto Nacional de Estadística y Censos [INEC], 2014). En el 2009, un estudio nacional 
encontró que aproximadamente el $2 \%$ de la población tenía una discapacidad (Misión Solidaria Manuela Espejo, 2012). El Consejo Nacional para la Igualdad de Discapacidades (2017) informó que hasta octubre del 2017 el 2,64\% de la población, equivalente a 429.475 individuos sobre una población de 16.221.610 ecuatorianos, se encontraba en el registro nacional de discapacidades. El cuadro 1 muestra esta información desglosada por género, tipo de discapacidad, grado de discapacidad y grupo etario. No obstante, las cifras en Ecuador serían de las más bajas de la región, en comparación con: 5,2\% en Perú (Instituto Nacional de Estadística e Informática, 2015), 6.3\% en Colombia (Observatorio Nacional de Discapacidad, 2014), 12,9\% en Argentina (INDEC, 2014), 16,7\% en Chile (Servicio Nacional de la Discapacidad, 2016) y 23,9\% en Brasil (Secretaria Nacional de Promoção dos Direitos da Pessoa com Deficiência, 2012).

Cuadro 1. Distribución relativa de personas con discapacidad por género, tipo, grado y grupo etario en Ecuador

\begin{tabular}{llllllll}
\hline \multicolumn{2}{c}{ TIPO } & \multicolumn{2}{c}{ GRADO } & \multicolumn{2}{c}{ GRUPO ETARIO } & \multicolumn{2}{c}{ GÉNERO } \\
\hline Física & $46,78 \%$ & $30 \%$ a $49 \%$ & $45,42 \%$ & O a 17 años & $13,54 \%$ & Masculino & $56,22 \%$ \\
Intelectual & $22,54 \%$ & $50 \%$ a $74 \%$ & $35,07 \%$ & 18 a 29 años & $14,86 \%$ & Femenino & $43,77 \%$ \\
Auditiva & $12,87 \%$ & $75 \%$ a $84 \%$ & $13,64 \%$ & 30 a 65 años & $48,21 \%$ & GLBTI* & $0,00 \%$ \\
Visual & $11,85 \%$ & $85 \%$ a $100 \%$ & $5,87 \%$ & 65 años o más & $23,40 \%$ & & \\
Psicosocial & $4,65 \%$ & & & & & \\
Lenguaje & $1,31 \%$ & & & & \\
\hline
\end{tabular}

\subsection{De la exclusión a la inclusión: La evolución sociohistórica de la discapacidad en la educación}

La discapacidad, lejos de su conceptualización técnica, es un fenómeno sociohistórico con una accidentada evolución. En un inicio, las personas con discapacidad, al igual que los enfermos mentales y los homosexuales, sufrieron de persecución, ostracismo y menosprecio. Su identidad histórica fue marcada por la exclusión, los prejuicios y los estereotipos de un pasado latente que han hecho de la discriminación y el aislamiento una constante (Baquero, 2015). Incluso el concepto de discapacidad en sí es novedoso, pues por la mayor parte de la historia se los llamó personas con defectos físicos, inválidos, minusválidos o deficientes (Samaniego, 2009). Evidentemente, en cada etapa histórica se han configurado formas particulares de concebir esta condición, por lo tanto, es posible sintetizar la visión y tratamiento que se dio a este grupo en tres estadios conceptuales: el modelo de prescindencia, el modelo rehabilitador y el modelo social (Palacios, 2008; Stang, 2011).

El primer modelo está gravemente influenciado por motivos religiosos, pues la discapacidad y otras condiciones eran vistas como consecuencia divina, un castigo proveniente de un ser superior. En este primer estadio, conocido como el modelo caritativo, el individuo con discapacidad es objeto de lástima y conmiseración que, bajo el marco de los ideales judeocristianos, debe ser auxiliado y asistido (Cruz y Casillas, 2017). Así, las personas con discapacidad son despojadas de su valía y autonomía personal. Pasan a ser vistas como prescindibles en la sociedad puesto que se los considera inhábiles para producir (Palacios, 2008). La respuesta usual ante la discapacidad era la marginación, internamiento y reclusión forzada, ignorando aspectos cruciales como la educación e inserción laboral (Baquero, 2015). 
En el segundo modelo, la persona discapacitada es vista desde una óptica médico-científica, analizada a partir de su disfunción orgánica. El quid de la cuestión pasa a ser la rehabilitación. Así la discapacidad se desembaraza de sus connotaciones religiosas para ser circunscrita en la lógica del déficit y el síntoma, donde es entendida en términos de normalidad-anormalidad, una desviación del estado "natural" del ser humano (Baquero, 2015). El modelo rehabilitador o médico-asistencialista, influenciado por el zeitgeist positivista de la época, pretende ocultar o desaparecer la diferencia con el fin de normalizar al individuo (Palacios, 2008). Bajo esta dialéctica, surgen las primeras iniciativas educacionales con los franceses L'Épée y Haüy, quienes fundaron las primeras escuelas especializadas para sordos e invidentes, respectivamente (Aquino, García e Izquierdo, 2012). Así se aprovechó la ciencia para consolidar las bases ideológicas de la exclusión.

Después de un extenso recorrido histórico, llegaría el modelo social. El individuo por fin es visto no como objeto de caridad o cuerpo deficiente, sino como un sujeto de derechos con capacidad para aportar a la sociedad. Este modelo apunta a potenciar la dignidad humana, la igualdad, la equidad, la autonomía y sobre todo la inclusión (Palacios, 2008). El advenimiento de estos nuevos ideales sacudió las bases epistémicas de la educación especial, que pasó a ser vista como un acto de segregación en sí mismo, pues excluía a las personas con discapacidad de la escolaridad regular debido a su condición (Aquino, García e Izquierdo, 2012; Samaniego, 2009). La inclusión viene a dar respuesta a los procesos de discriminación institucionalizados y se sitúa como un nuevo paradigma en la educación.

En vez de concentrarse en cómo integrar a este grupo de individuos a los procesos de educación estándar, el enfoque inclusivo se preocupa por transformar el sistema educativo para responder a la diversidad, de forma que esta sea vista como una oportunidad de enriquecimiento y no como una contrariedad (Booth, 2003). A partir de este eje de acción, se procura abandonar aquella construcción narrativa excluyente que se fue gestando a través del tiempo y sustituirla por una nueva, fundada en el respeto y los derechos esenciales. Es en este punto de inflexión que la persona con discapacidad deja de acomodarse forzosamente a un sistema que segrega y es este último que empieza a adaptarse a sus necesidades.

Desde entonces, el concepto se ha expandido para englobar los ideales de equidad, diversidad y participación (Payà, 2010). La inclusión es ahora un proceso que busca dar solución a la diversidad de necesidades presentes en el entorno educativo mediante la modificación de los contenidos, el abordaje, las estructuras y estrategias (Booth, 2003). En definitiva, la óptica contemporánea da mayor importancia a las necesidades del individuo para involucrarse plenamente en su entorno que a la condición que lo aqueja. Es decir, la inclusión apunta a brindar ayudas técnicas o pedagógicas para que el estudiante logre sus fines educativos. También resta protagonismo a la condición y se concentran los esfuerzos en cumplir con determinadas necesidades que permitan al sujeto empoderarse de su situación.

El término inclusión apareció por primera vez en el contexto internacional durante la Convención Mundial de Jomtien en 1990, escenario de la Educación para Todos (Payà, 2010). A raíz de esto surgieron una serie de ordenamientos, tratados y acuerdos que pretendían reducir la discriminación y motivar la inclusión: Normas Uniformes sobre la Igualdad de Oportunidades para las Personas con Discapacidad, por las Naciones Unidas en 1993; la Declaración de Salamanca en la Conferencia Mundial sobre Necesidades Educacionales Especiales, por la UNESCO en 1994; la Convención Interamericana para la 
Eliminación de Todas las Formas de Discriminación contra las Personas Portadoras de Deficiencia en 1999; y por último, la Convención sobre los derechos de las personas con discapacidad, por las Naciones Unidas en 2006 (Crosso, 2010). Además, algunas organizaciones expidieron normativas, protocolos y manuales que pretendían estandarizar el abordaje de la discapacidad a nivel mundial, entre ellas la Clasificación Internacional del Funcionamiento, de la Discapacidad y de la Salud o CIF (OMS, 2001).

De esta forma, la sociedad asumió el reto de la inclusión educativa "que reconozca la diferencia y problematice la enseñanza”, no viceversa (Baquero, 2015, p. 176). La atención internacional que recibió el proyecto impulsó reformas en varias regiones incluida Latinoamérica y propició la adopción de un enfoque centrado en los derechos, propio del modelo social, en más del 50\% de países de esa región (Comisión Económica para América Latina y el Caribe, 2014; Fajardo, 2017; Samaniego, 2009). No obstante, uno de los hitos más valiosos de esta última década ha sido generar un sentimiento de interés generalizado que ha devenido en proyectos, investigación, iniciativas de inclusión particular y la toma de consciencia sobre la tragedia sociohistórica de la exclusión.

El impacto del movimiento pro-inclusión no puede ser subestimado. Las estadísticas en los años posteriores demostrarían este progreso. La OMS (2011) reportó que la proporción estimada de personas con discapacidad que han completado la educación primaria, a nivel global, es del 53,2\%, en comparación al 67,4\% de personas sin discapacidad, lo cual señala una brecha decreciente. En Estados Unidos el número de niños y jóvenes con discapacidad servidos bajo la Individuals with Disabilities Education Act (IDEA, por sus siglas en inglés) ha aumentado progresivamente desde 1976 hasta 2017 (National Center for Education Statistics, 2016). En Ecuador, se estima que el 78\% de niños y jóvenes con discapacidad entre los 3 y 17 años de edad asiste al sistema educativo ${ }^{1}$, lo cual implica un avance loable (Ministerio de Educación, 2017). No obstante, pese al progreso a nivel primario y secundario, la inclusión no ha alcanzado al sistema de educación superior.

Durante siglos, la universidad fue un espacio altamente excluyente que servía a una población mayormente homogénea: varones heterosexuales, blancos y de estratos socioeconómicos altos. La modernidad trajo consigo una profunda recomposición de la población universitaria, permitiendo el acceso a grupos históricamente marginados desde las mujeres y los afrodescendientes hasta las personas con discapacidad (Cruz y Casillas, 2017). No obstante, aún quedan vestigios remanentes de la época de exclusión, óbices que dificultan el ingreso, permanencia y egreso de estos colectivos. Varios autores han señalado que uno de los escollos clave que enfrenta la persona con discapacidad son las barreras actitudinales, entre ellas la percepción de su condición y el estigma asociado a ella (APA, 2009; Castignani et al., 2014).

Las consecuencias de estos impedimentos son evidentes en las estadísticas de ingreso y egreso de estudiantes con discapacidad a determinado tipo de carreras. Durante la última década, autores como Kahn y Ginther (2017) han denunciado la histórica infrarrepresentación de las mujeres en las carreras CTIM, acrónimo utilizado para designar las disciplinas académicas de ciencia, tecnología, ingeniería y matemáticas. Este mismo fenómeno se repite para las personas con discapacidad. En Estados Unidos, dicho colectivo solo compone el $10 \%$ de estudiantes de pregrado, el $5 \%$ de posgrado, el $1 \%$ de doctorado

\footnotetext{
${ }^{1}$ La información procede de una rueda de prensa del ministro de educación, publicada en la página del Ministerio de Educación, pero no consta en ningún documento oficial.
} 
y el $2 \%$ de la población laboral de dichas carreras (Moon et al., 2012). En cambio, las personas con discapacidad se han concentrado en otras áreas, como la psicología, en la que representan el 23,1\% de los recipientes doctorales, siendo este el porcentaje más alto entre las ciencias (APA, 2009).

\subsection{La discapacidad: Un reto para la educación superior ecuatoriana del siglo XXI}

Según Peralta (2007), las personas con discapacidad constituyen menos del $1 \%$ de la población universitaria mundial, sugiriendo que aún persisten muchos obstáculos que impiden su acceso a este nivel de educación. Lissi y otros (2009) advierte que en Chile solo el 6,6\% de los jóvenes con discapacidad ingresa a la universidad y que la tasa de egreso es incluso menor. En Estados Unidos, la proporción de estudiantes de pregrado con discapacidades pasó de aproximadamente $6 \%$ en 1999 a $11 \%$ de todos los estudiantes universitarios del 2012; sin embargo, sólo el 34\% de ellos son capaces de completar su titulación tras 8 años (Pérez, Gulley y Prickett, 2016). En España, aunque los jóvenes con discapacidad representan entre el $2 \%$ y el $2,5 \%$ de la población juvenil general, sólo componen el 0,5\% del estudiantado universitario (Peralta, 2007).

En el 2004, pese al andamiaje legal que amparaba y promovía la inclusión en Ecuador, el $37,9 \%$ de la población con discapacidad no había recibido educación formal alguna y apenas el 1,8\% había tenido acceso a la educación superior, de los cuales el 62,9\% eran varones (Rodríguez, 2004). En el año 2009, un estudio representativo a nivel nacional encargado a la Misión Solidaria Manuela Espejo (2012) concluyó que solo el 1,9\% de dicho colectivo había alcanzado el grado universitario. Según una nota de prensa, en el 2015 alrededor de 5.053 personas con discapacidad, equivalente al 1,2\% del total en ese entonces, asistían a una institución de educación superior (Ministerio de Salud Pública, 2015; Redacción Sociedad, 2015). Desde entonces, la información acerca de la prevalencia de personas con discapacidad en las instituciones de educación superior ecuatorianas es virtualmente nula ${ }^{2}$.

En el año lectivo 2011-2012, la Universidad Técnica Salesiana y la Universidad Laica Vicente Rocafuerte de Guayaquil optaron por publicar un informe con estadísticas referentes a sus planteles, reportando la tenencia de 370 y 7 estudiantes con discapacidad respectivamente (Bodero y Terranova, 2012; Gallegos, 2011). No obstante, ambas investigaciones comparten graves limitaciones. La primera describe minuciosamente las acciones, dificultades y logros de la institución en torno a la inclusión; sin embargo, se limita a comunicar el número de estudiantes con discapacidad en una única cohorte, imposibilitando un análisis histórico del ingreso y egreso de estos y la evaluación real de las medidas reportadas (Gallegos, 2011). La segunda, a más de socializar la cantidad de estudiantes con discapacidad en el periodo mencionado, hace alusión a cuatro entrevistas de las cuales solo presenta brevemente una y a modo anecdótico.

Otras instituciones, como la Universidad Metropolitana del Ecuador y la Facultad Latinoamericana de Ciencias Sociales, también han publicado investigaciones al respecto. La primera de ellas propone un modelo "para la gestión del proceso de acceso y retención de estudiantes universitarios con discapacidad"; sin embargo, no presenta evidencia empírica de la puesta en marcha del mismo (Espinosa, Gómez y Cañedo, 2012, p. 255).

\footnotetext{
${ }^{2}$ El autor solicitó esta información al Consejo Nacional para la Igualdad de Discapacidades y a la Secretaría de Educación Superior, Ciencia, Tecnología e Innovación por los medios oficiales. El primer organismo remitió al segundo y este último informó que no existe una base de datos al respecto, pero que sería posible solicitar dicha información en cada una de las universidades del país.
} 
Mientras tanto, la segunda se propone visibilizar su trayectoria en torno a la inclusión a través de entrevistas, pero tampoco presenta pruebas concretas de algún progreso alcanzado (Esteves y Santos, 2014). La falta de información fiable y consistente es una problemática a nivel nacional y regional que imposibilita un diagnóstico preciso de la situación, dificultando la elaboración de planes e iniciativas de solución (Cueva et al., 2017; Espinosa, Gómez y Cañedo, 2012; Herrera et al., 2018; Stang, 2011).

Es menester de las universidades no solo acoger a este grupo, sino brindarles una educación competente y para ello es necesario que se investigue, sin tapujos, la discriminación (Aquino, García e Izquierdo, 2012). Sin un abordaje concreto y efectivo de la exclusión en la educación superior, se seguirán consolidando las desventajas sociales y económicas que históricamente han sufrido las personas con discapacidad. Pese a que en los últimos años el número de publicaciones con respecto a la discapacidad en la educación superior ecuatoriana ha incrementado exponencialmente, la escasez de información concreta e investigaciones profundas sigue siendo palpable (Cueva et al., 2017; Espinosa y Goetschel, 2014; Martínez, Silva y Naranjo, 2016; Vizñay, Campoverde y Poma, 2017).

Fomentar la inclusión no es una gracia del Estado ecuatoriano o las instituciones educativas del mismo país, sino una responsabilidad ético-jurídica de ambos. Así, la inclusión educativa en Ecuador está avalada por la Carta Suprema, la Ley Orgánica de Discapacidades (Asamblea Nacional de la República del Ecuador, 2012), la Ley Orgánica de Educación Intercultural (Asamblea Nacional de la República del Ecuador, 2011), la Ley Orgánica de Educación Superior (Asamblea Nacional de la República del Ecuador, 2010), el Plan Nacional del Buen Vivir (Secretaría Nacional de Planificación y Desarrollo, 2017), la Convención Internacional sobre los Derechos de las Personas con Discapacidad (Naciones Unidas, 2006) e incontables acuerdos ministeriales (Ministerio de Educación, 2013). Inclusive, varias universidades contemplan este aspecto como parte integral de sus políticas internas.

Por ejemplo, la Universidad Católica de Santiago de Guayaquil (en adelante UCSG) en su Plan Estratégico de Desarrollo Institucional se propone desarrollar "acciones que posibiliten el ingreso y la permanencia de estudiantes con discapacidad" e "integrar y socializar a los estudiantes con discapacidad" (UCSG, 2017). Pero la consecución de estos encomiables objetivos no es sola responsabilidad de la institución, como entidad legal, sino también de la comunidad que la compone, como los docentes. Estos últimos han encabezado algunos de los proyectos de inclusión educativa más relevantes, como la digitalización y acondicionamiento de materiales bibliográficos para su lectura por medio de programas de texto a voz y la elaboración de maquetas tridimensionales de la anatomía cerebral, apoyos provechosos para el importante grupo de estudiantes con discapacidad visual en el plantel.

Ciertamente, las disposiciones gubernamentales han tenido un rol importante en este movimiento a favor de la inclusión en la educación superior; no obstante, algunas de las medidas impuestas desde el Estado han sido inefectivas y en algunos casos contraproducentes. El autor del presente concuerda con Espinosa, Gómez y Cañedo (2012) en que los actuales modelos nacionales de evaluación de las instituciones de educación superior se centran en indicadores técnicos como el acondicionamiento arquitectónico, la ayuda financiera y la asignación de cupos prestados al colectivo en cuestión; pero dejan de lado aspectos cruciales, de índole cualitativa, como el acceso a la información, las metodologías de enseñanza y evaluación o la preparación docente (Bell, 
2017). Esto hace de la inclusión una mera lista de requisitos por cumplir y no un proceso sostenido de mejora, refinamiento y reelaboración de la experiencia educativa a favor de los estudiantes.

En México, un estudio reciente concluyó que de 53 universidades solo 12 reportaron la existencia de programas, iniciativas o acuerdos para lograr una mayor inclusión educativa (Cruz y Casillas, 2017). Aunque no existan investigaciones similares en Ecuador, una revisión profunda del estado del arte sugiere que el panorama puede ser igual o incluso menos favorable. Sin investigaciones sólidas y actuales, coordinar iniciativas de intervención puede ser ineficiente y poco adecuado. Es necesario abordar la falta de información e investigación para así lograr un diagnóstico fiable de la situación que permita la toma de acción.

\section{Metodología}

Esta investigación es no-experimental porque no cumple con los criterios de manipulación de variables, y descriptiva, ya que detalla las propiedades del fenómeno como se presenta (Hernández-Sampieri, Fernández y Baptista, 2010). Además, su alcance es exploratoriodescriptivo porque examina un problema poco estudiado en un contexto particular y pretende detallar sus propiedades, siendo esta la primera investigación ecuatoriana que se interroga por el interés de una comunidad académica en el tema de las discapacidades y analiza las características del estudiantado con discapacidad de una universidad particular.

En primer lugar, se presenta un estudio descriptivo de las investigaciones sobre discapacidad realizadas por los miembros de la UCSG. Para esto, se designa como fuente el Repositorio Digital Institucional (en adelante RDI) ya que contiene el mayor número de investigaciones realizadas por los estudiantes de pregrado, estudiantes de máster y profesores de la institución. Por lo tanto, se considera que refleja el interés científico de la comunidad académica de la universidad en cuestión.

Utilizando el formulario de búsqueda se empleó únicamente la categoría discapacidad, término que la Convención Internacional por los Derechos de las Personas con Discapacidad (2008) estimó adecuado y es considerado correcto a nivel global. En la estrategia de revisión se incluyeron todas las publicaciones del RDI. La búsqueda fue realizada por el autor y una investigadora independiente, lo cual permitió cotejar los resultados y garantizar la máxima consistencia, alcanzando una correspondencia del 100\% entre ambas búsquedas. En los resultados se detalla la proporción de publicaciones sobre discapacidad en relación al total y el porcentaje de publicaciones sobre discapacidad por año, carrera y facultad.

En segundo lugar, se presenta un análisis estadístico de los estudiantes con discapacidad en la institución. Para ello, se utiliza como fuente de información dos conjuntos de datos proporcionados por el Departamento de Bienestar Estudiantil de la institución. El primero corresponde al listado de estudiantes que se identificaron como personas con discapacidad en el sistema institucional, por vía digital. El segundo es el reporte de estudiantes becados por discapacidad. Ambos registros corresponden únicamente a estudiantes de pregrado matriculados entre el 2013 y 2017.

En el análisis se detalla el número de estudiantes con discapacidad distribuidos por grado de discapacidad, tipo de discapacidad, carrera, facultad, así como la prevalencia de estudiantes con discapacidad con respecto a la población general estudiantil por año. Los 
datos procedentes del RDI y del Departamento de Bienestar Estudiantil se introdujeron por separado en hojas de cálculo del programa Excel 2016 y fueron, posteriormente, traspasadas al programa SPSS Statistics 20. Se utilizaron ambas herramientas para la elaboración de las tablas de distribución y se generaron las gráficas.

\section{Resultados}

Los resultados se dividen en dos secciones. La primera corresponde al análisis estadístico de las publicaciones sobre discapacidad de la UCSG y la segunda corresponde al análisis estadístico de estudiantes con discapacidad matriculados desde el año 2013 al 2017 en la misma institución.

\subsection{Estudio descriptivo de las publicaciones sobre discapacidad de la Universidad Católica de Santiago de Guayaquil}

El RDI almacena 9.483 trabajos de investigación publicados desde el 2000 hasta el presente, exceptuando una de 1986 . El 86,5\% (8.202) pertenece a carreras de pregrado y el 13,5\% (1.281) restantes, a posgrados. La búsqueda arrojó 129 resultados, equivalente al $1,36 \%$ del total de publicaciones. Su año de publicación varía desde el 2010 hasta el 2017. Las publicaciones pertenecen a veintisiete carreras distintas y ocho maestrías. El cuadro 2 presenta la distribución absoluta de las publicaciones según el año de publicación, carrera y facultad.

El 92,2\% (119) de las investigaciones con la categoría discapacidad pertenecen a las carreras de pregrado. La carrera con el mayor porcentaje de investigaciones es Terapia Física (10\%), seguida por Medicina (8,5\%) y Administración de Empresas Turísticas y Hoteleras $(8,5 \%)$. La facultad con el mayor porcentaje de publicaciones es la de Ciencias Médicas (28,6\%), seguida por la de Arquitectura y Diseño $(15,5 \%)$ y la de Especialidades Empresariales (14,7\%). En la otra mano, la Facultad de Educación Técnica para el Desarrollo (2,3\%) y de Ciencias Económicas y Administrativas (1,5\%) tienen el menor porcentaje de investigaciones publicadas al respecto. Hasta la fecha, la Facultad de Ingeniería no ha publicado trabajos en la categoría estudiada. Los resultados indican que las carreras de corte social y de la salud tienen un número mayor de investigaciones que aquellas de corte técnico.

Por otra parte, el año con el mayor porcentaje de investigaciones fue el 2016 (32,5\%), seguido del $2017(23,2 \%)$ y el $2014(17,8 \%)$. Los resultados indican que el número de publicaciones con la categoría propuesta ha ido en aumento a través de los años, aunque de forma inconsistente. También sugieren que existe un interés creciente en la temática, especialmente en los estudiantes de pregrado. La figura 1 muestra la distribución absoluta de publicaciones por año, incluyendo una previsión hasta el 2020, elaborada a partir de un algoritmo de suavización exponencial con un intervalo de confianza al 95\%. 
Cuadro 2. Distribución absoluta de publicaciones por año de publicación, carrera y facultad

\begin{tabular}{|c|c|c|c|c|c|c|c|c|c|c|}
\hline FACULTAD & CARRERA* & $\stackrel{\circ}{\circ}$ & ఫ) & \& & $\stackrel{\infty}{\circ}$ & 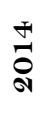 & $\frac{19}{8}$ & $\stackrel{0}{0}$ & $\stackrel{-}{a}$ & 离 \\
\hline \multirow{3}{*}{$\begin{array}{l}\text { Arquitectura y } \\
\text { Diseño }\end{array}$} & Arquitectura & & & & & 3 & 2 & & & 5 \\
\hline & Diseño de Interiores & & & 1 & & & & 3 & 3 & 7 \\
\hline & Gestión Gráfica Publicitaria & & & & & 1 & 3 & 1 & 3 & 8 \\
\hline \multirow{5}{*}{$\begin{array}{l}\text { Artes y } \\
\text { Humanidades }\end{array}$} & Ing. en Producción y & & & & & & & & & \\
\hline & Dirección de Artes & & & & 1 & 4 & 1 & & & 6 \\
\hline & Multimedia & & & & & & & & & \\
\hline & Ing. en Producción y & & & & & & & & & \\
\hline & $\begin{array}{l}\text { Dirección en Artes } \\
\text { Audiovisuales }\end{array}$ & & & & & & 2 & 1 & & 3 \\
\hline \multirow{2}{*}{$\begin{array}{l}\text { Ciencias Económicas } \\
\text { y Administrativas }\end{array}$} & Administración de Empresas & & & & & & & 1 & & 1 \\
\hline & Economía & & & & & & 1 & & & 1 \\
\hline \multirow{5}{*}{ Ciencias Médicas } & Enfermería & & & & 1 & 1 & & 5 & 1 & 8 \\
\hline & Medicina & & 1 & & 1 & 1 & & 5 & 3 & 11 \\
\hline & Nutrición, Dietética y Estética & 2 & & 1 & & & & 1 & & 4 \\
\hline & Odontología & & & & & & & 1 & & 1 \\
\hline & Terapia Física & & & & & 1 & 2 & 8 & 2 & 13 \\
\hline \multirow{3}{*}{$\begin{array}{l}\text { Educación Técnica } \\
\text { para el Desarrollo }\end{array}$} & $\begin{array}{l}\text { Ingeniería Electrónica en } \\
\text { Control y Automatismo }\end{array}$ & & & & & 1 & & & & 1 \\
\hline & Ingeniería en Electromecánica & & & & & 1 & & & & 1 \\
\hline & $\begin{array}{l}\text { Ingeniería en } \\
\text { Telecomunicaciones } \\
\end{array}$ & & & & & & & & 1 & 1 \\
\hline \multirow{8}{*}{$\begin{array}{l}\text { Especialidades } \\
\text { empresariales }\end{array}$} & Administración de Empresas & & & & & & & & & \\
\hline & $\begin{array}{l}\text { Turísticas y Hoteleras } \\
\text { Bilingüe }\end{array}$ & & & & 1 & 1 & 1 & 5 & 3 & 11 \\
\hline & Administración de Empresas & & & & & & & & & \\
\hline & $\begin{array}{l}\text { Turísticas y Hoteleras } \\
\text { Bilingüe - Distancia }\end{array}$ & & & & & & & & 1 & 1 \\
\hline & Administración de Ventas & & & & & 2 & & & & 2 \\
\hline & Emprendimiento & & & & & & & & 1 & 1 \\
\hline & Marketing & & & & & & & & 1 & 1 \\
\hline & Marketing - Distancia & & & & & 1 & & 1 & & 2 \\
\hline \multirow{4}{*}{$\begin{array}{l}\text { Filosofía, Letras y } \\
\text { Ciencias de la } \\
\text { Educación }\end{array}$} & Comunicación social & & & & & & 1 & & 1 & 2 \\
\hline & Pedagogía & & & & & 2 & & 1 & 1 & 4 \\
\hline & Psicología Clínica & & 1 & 1 & 1 & & 3 & 3 & & 9 \\
\hline & Psicología Organizacional & & & & & & 2 & & & 2 \\
\hline \multirow{2}{*}{ Jurisprudencia } & Trabajo Social & & & & & 3 & & 1 & 5 & 9 \\
\hline & Derecho - distancia & & & & & & & 2 & 1 & 3 \\
\hline \multirow{6}{*}{$\begin{array}{l}\text { Sistema de } \\
\text { Posgrados }\end{array}$} & $\begin{array}{l}\text { En Administración de } \\
\text { Empresas }\end{array}$ & & & & 2 & & & & & 2 \\
\hline & En Derecho Constitucional & & & & & & & 2 & & 2 \\
\hline & $\begin{array}{l}\text { En Derecho Notarial y } \\
\text { Registral }\end{array}$ & & & & & & & & 1 & 1 \\
\hline & En Educación Superior & & & & & & & & 1 & 1 \\
\hline & $\begin{array}{l}\text { En Gerencia en Servicios de la } \\
\text { Salud }\end{array}$ & & & & & 1 & & 1 & & 2 \\
\hline & En Telecomunicaciones & & & & & & & & 1 & 1 \\
\hline \multirow{2}{*}{$\begin{array}{l}\text { Sistema de } \\
\text { Posgrados: } \\
\text { Especialización }\end{array}$} & $\begin{array}{l}\text { En Anestesiología y } \\
\text { Reanimación }\end{array}$ & & & & & & 1 & & & 1 \\
\hline & En Medicina Interna & & & 1 & & & & & & 1 \\
\hline Total & & 2 & 2 & 4 & 7 & 23 & 19 & 42 & 30 & 129 \\
\hline
\end{tabular}

Nota: *Los nombres de algunas carreras han sido actualizados.

Fuente: Elaboración propia. 


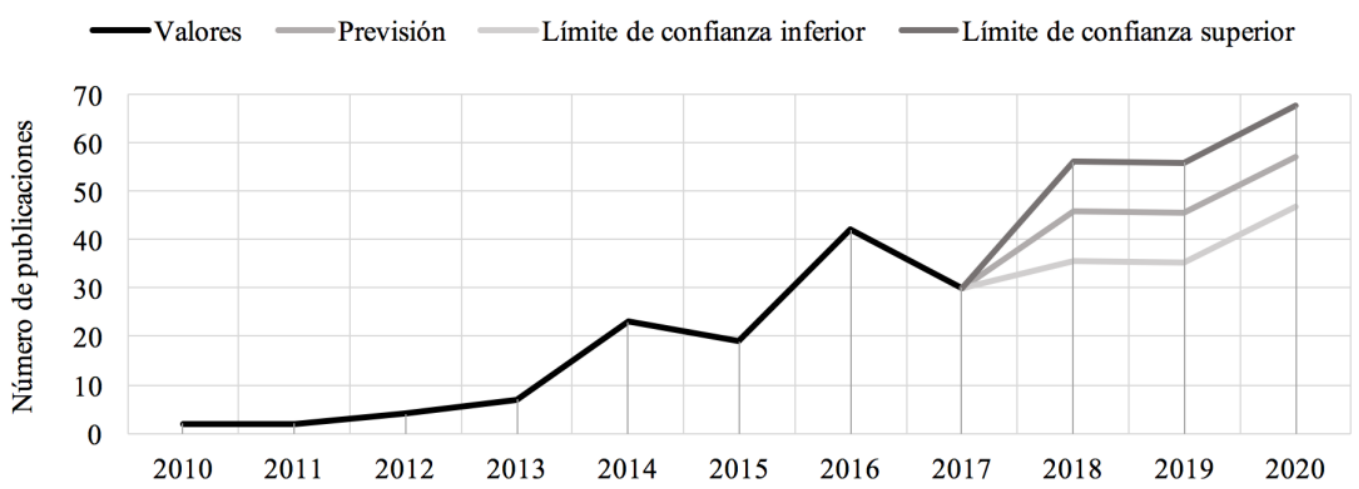

Figura 1. Distribución absoluta de publicaciones por años (2010-2020)

Fuente: Elaboración propia.

\subsection{Análisis de los estudiantes con discapacidad en la Universidad Católica de Santiago de Guayaquil}

La data proporcionada por el Departamento de Bienestar Estudiantil de la UCSG consta de dos conjuntos, el primero de 762 registros y el segundo de 480 registros. Ambos reportes fueron unificados en una única matriz para lograr mayor completitud de los datos. El número de registros únicos, sin duplicados por número de identificación nacional, son 195. De dicho número, tres fueron excluidos por reconocer porcentajes de discapacidad menores a $4 \%$ que, con base en la CIF, no califica como discapacidad. Se puede afirmar que entre el año 2013 y el 2017 se matricularon en la institución al menos 192 personas con una discapacidad autoidentificada y/o convalidada por el Departamento de Bienestar Estudiantil.

El 51,5\% (99) es de género masculino, indicando que no existe una diferencia considerable entre géneros. Este resultado contrasta con la tendencia reportada en años anteriores. El $73,4 \%$ (141) es originario de la provincia donde se encuentra la institución y el 26,6\% (51) restante, de otras provincias del país, lo cual concuerda casi perfectamente con el porcentaje general de estudiantes interprovinciales de la institución (27\%). Por último, todos cuentan con un número de identificación nacional válido por lo cual se determina que no hay estudiantes con discapacidad extranjeros.

El 85\% (165) ha recibido ayuda financiera al menos una vez, indicando que el 15\% (27) restante no ha solicitado dicho apoyo. Con base en los parámetros nacionales, los estudiantes con discapacidad pueden clasificarse en: 82 estudiantes pertenecen a la categoría de $30 \%$ a $49 \%$; 49 a la categoría de $50 \%$ a $74 \% ; 11$ a la categoría de $75 \%$ a $84 \%$; 6 a la categoría de $85 \%$ a $100 \%$; 7 tienen un grado menor a $29 \%$, por lo cual no cumplen los parámetros de los organismos nacionales pertinentes; y 37 no han reportado su grado de discapacidad. La figura 2 presenta la distribución relativa de los estudiantes con discapacidad según el grado. 


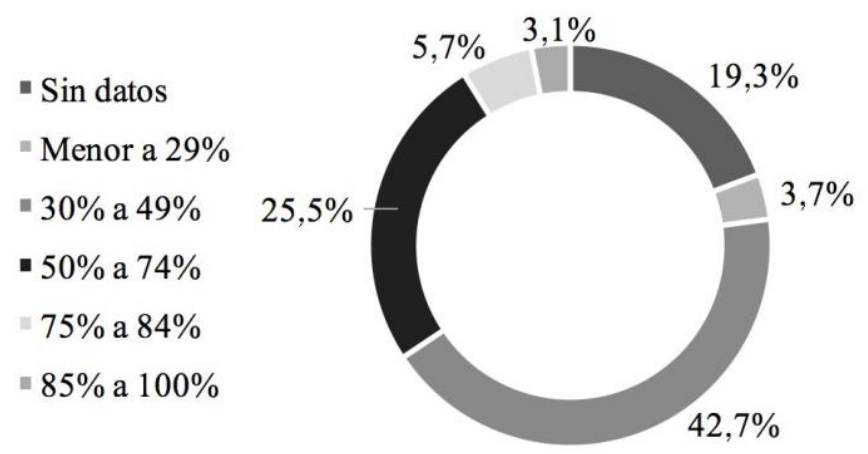

Figura 2. Distribución relativa de estudiantes con discapacidad según el grado Fuente: Elaboración propia.

La carrera que ha tenido el mayor porcentaje de estudiantes con discapacidad en el tiempo señalado es Derecho (20,3\%), seguida por Trabajo Social (9,8\%), Enfermería (5,7\%) y Medicina $(5,2 \%)$. La facultad con mayor prevalencia de estudiantes con discapacidad es la Facultad de Jurisprudencia (30,2\%), seguida por la Facultad de Ciencias Médicas (18,2\%) y la Facultad de Especialidades Empresariales (13,5\%). Conforme a la literatura, las carreras de corte CTIM, como aquellas de la Facultad de Educación Técnica para el Desarrollo y Facultad de Ingeniería presentan un bajo porcentaje de estudiantes con discapacidad en comparación a las de corte social o humanístico. Por otra parte, Psicología Clínica y Psicología Organizacional no presentaron índices especialmente altos, contrario a lo que reportaron investigaciones extranjeras.

El tipo de discapacidad más frecuente es la discapacidad física $(37,5 \%)$, seguida por la visual $(18,2 \%)$ y la auditiva $(16,6 \%)$. Por otra parte, los tipos menos representados son la discapacidad psicosocial $(4,6 \%)$ y lingüística (1\%). Estos resultados concuerdan parcialmente con los porcentajes nacionales por tipo de discapacidad, especialmente en la representación de personas con discapacidad física, visual y auditiva. Sin embargo, contrastan en el caso de la discapacidad intelectual, la cual representa un 5,7\% del estudiantado con discapacidad, pero es la segunda con mayor prevalencia a nivel nacional. También cabe señalar la infrarrepresentación de la discapacidad lingüística. El cuadro 3 presenta la distribución absoluta de los estudiantes con discapacidad por tipo de discapacidad, carrera y facultad.

Por otra parte, el número de estudiantes matriculados con discapacidad ha incrementado desde el año 2013 hasta el 2017 en una tasa de crecimiento anual de 9,7\%. Desde el 2013 hasta el 2017, el porcentaje anual de estudiantes con discapacidad ha incrementado progresivamente desde el $0,47 \%$ hasta componer el $0,73 \%$ del total de estudiantes matriculados en la institución. El cuadro 4 presenta la prevalencia de estudiantes con discapacidad en comparación a la población estudiantil general desde el 2013 al 2017. La prevalencia de estudiantes con discapacidad ha sido calculada a partir del número de estudiantes con discapacidad sobre el número de estudiantes por año. 
Cuadro 3. Distribución absoluta de estudiantes con discapacidad por tipo de discapacidad, carrera y facultad

\begin{tabular}{|c|c|c|c|c|c|c|c|c|c|}
\hline FACULTAD & CARRERA & 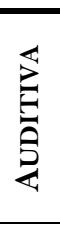 & $\underset{\widetilde{U}}{\mathbb{n}}$ & 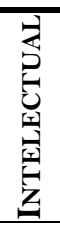 & 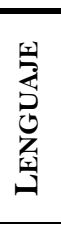 & 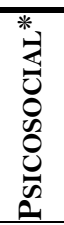 & 空 & 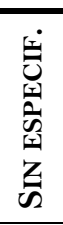 & 逍 \\
\hline \multirow{2}{*}{ Arquitectura y diseño } & Arquitectura & 2 & 2 & & 1 & & & & 5 \\
\hline & Diseño de Interiores & 1 & & & & & & & 1 \\
\hline \multirow{3}{*}{ Artes y Humanidades } & $\begin{array}{l}\text { Ing. en Producción y Dirección } \\
\text { de Artes Multimedia }\end{array}$ & 1 & 1 & & & & & & 2 \\
\hline & $\begin{array}{l}\text { Ing. en Producción y Dirección } \\
\text { en Artes Audiovisuales }\end{array}$ & 1 & 2 & & & & & & 3 \\
\hline & Música & & & 1 & & 1 & 1 & & 3 \\
\hline \multirow{3}{*}{$\begin{array}{l}\text { Ciencias económicas y } \\
\text { administrativas }\end{array}$} & Administración de Empresas & & 3 & 2 & 1 & & 1 & 2 & 9 \\
\hline & Economía & & & 1 & & & 2 & & 3 \\
\hline & Contabilidad y Auditoría & & 2 & & & & & & 2 \\
\hline \multirow{5}{*}{ Ciencias médicas } & Enfermería & 4 & 4 & & & & 3 & & 11 \\
\hline & Medicina & 1 & 6 & & & 1 & 1 & 1 & 10 \\
\hline & Nutrición, Dietética y Estética & 1 & 3 & & & & & 1 & 5 \\
\hline & Odontología & 1 & 2 & & & & 1 & & 4 \\
\hline & Terapia Física & & 1 & & & 2 & 1 & 1 & 5 \\
\hline \multirow{5}{*}{$\begin{array}{l}\text { Educación Técnica } \\
\text { para el Desarrollo }\end{array}$} & Ingeniería Agropecuaria & & & & & & & 1 & 1 \\
\hline & $\begin{array}{l}\text { Ingeniería Electrónica en } \\
\text { Control y Automatismo }\end{array}$ & 1 & 1 & & & & & & 2 \\
\hline & Ingeniería en Electromecánica & & 3 & & & & & & 3 \\
\hline & $\begin{array}{l}\text { Ingeniería en } \\
\text { Telecomunicaciones }\end{array}$ & & & & & & 1 & & 1 \\
\hline & $\begin{array}{l}\text { Medicina, Veterinaria y } \\
\text { Zootecnia }\end{array}$ & 1 & 1 & & & & & 1 & 3 \\
\hline \multirow{6}{*}{$\begin{array}{l}\text { Especialidades } \\
\text { empresariales }\end{array}$} & $\begin{array}{l}\text { Administración de Empresas } \\
\text { Turísticas y Hoteleras } \\
\text { Bilingüe }\end{array}$ & 1 & & 1 & & 1 & 1 & 1 & 5 \\
\hline & $\begin{array}{l}\text { Comercio y Finanzas } \\
\text { Internacionales }\end{array}$ & & 1 & & & & 3 & & 4 \\
\hline & Administración de Ventas & 1 & 3 & & & & 1 & 2 & 7 \\
\hline & Emprendimiento & & 1 & & & & 1 & & 2 \\
\hline & $\begin{array}{l}\text { Gestión Empresarial } \\
\text { Internacional }\end{array}$ & & 3 & & & & & & 3 \\
\hline & Marketing & 1 & 2 & & & & 1 & 1 & 5 \\
\hline \multirow{4}{*}{$\begin{array}{l}\text { Filosofía, Letras y } \\
\text { Ciencias de la } \\
\text { Educación }\end{array}$} & Comunicación social & 3 & 1 & 2 & & 1 & 2 & & 9 \\
\hline & Pedagogía & & & & & & 1 & & 1 \\
\hline & Psicología Clínica & 1 & 1 & & & & 4 & 1 & 7 \\
\hline & Psicología Organizacional & 1 & 1 & 1 & & & 1 & & 4 \\
\hline \multirow[b]{2}{*}{ Ingeniería } & Ingeniería Civil & 1 & 2 & & & & 1 & & 4 \\
\hline & $\begin{array}{l}\text { Ingeniería en Sistemas } \\
\text { Computacionales }\end{array}$ & & 3 & & & & 1 & & 4 \\
\hline \multirow{2}{*}{ Jurisprudencia } & Trabajo Social & 3 & 5 & 2 & & 1 & 2 & 6 & 19 \\
\hline & Derecho & 5 & 18 & 1 & & 2 & 5 & 8 & 39 \\
\hline Sin especificar & & & & & & & & 6 & 6 \\
\hline Total & & 31 & 72 & 11 & 2 & 9 & 35 & 32 & 192 \\
\hline
\end{tabular}

Nota: * Los tipos de discapacidad mental y psicológica, presentes en los registros han sido reunidos en el tipo psicosocial conforme a las disposiciones del Ministerio de Salud Pública.

Fuente: Elaboración propia. 
Cuadro 4. Proporción de estudiantes con discapacidad por año en comparación a la población estudiantil general

\begin{tabular}{|c|c|c|c|c|c|c|}
\hline \multirow[t]{2}{*}{ AÑo } & \multicolumn{3}{|c|}{$\begin{array}{c}\mathrm{N}^{\circ} \text { DE ESTUDIANTES } \\
\text { MATRICULADOS CON } \\
\text { DISCAPACIDAD } \\
\end{array}$} & \multirow{2}{*}{$\begin{array}{c}\mathrm{N}^{\circ} \text { DE } \\
\text { ESTUDIANTES } \\
\text { MATRICULADOS } \\
\text { POR AÑO }\end{array}$} & \multirow{2}{*}{$\begin{array}{c}\text { PROPORCIÓN } \\
\text { DE } \\
\text { ESTUDIANTES } \\
\text { CON } \\
\text { DISCAPACIDAD }\end{array}$} & \multirow{2}{*}{$\begin{array}{c}\text { TASA BÁSICA } \\
\text { DE } \\
\text { CRECIMIENTO }\end{array}$} \\
\hline & Semestre A & Semestre B & Únicos & & & \\
\hline 2013 & 58 & 59 & 71 & 15.192 & $0,47 \%$ & - \\
\hline 2014 & 60 & 81 & 89 & 19.472 & $0,46 \%$ & $-0,01 \%$ \\
\hline 2015 & 94 & 83 & 110 & 19.476 & $0,56 \%$ & $0,11 \%$ \\
\hline 2016 & 91 & 93 & 109 & 18.047 & $0,60 \%$ & $0,04 \%$ \\
\hline 2017 & 88 & 88 & 103 & $14.063^{*}$ & $0,73 \%$ & $0,13 \%$ \\
\hline
\end{tabular}

Nota: *Este dato está sujeto a cambios previo a la rendición oficial de cuentas.

Fuente: Elaboración propia.

\section{Conclusiones}

La mayoría de las investigaciones sobre discapacidad producidas por la comunidad académica de la institución pertenecen a las Ciencias Sociales y de la Salud. Por otra parte, la contribución de las ingenierías y ramas técnicas es ínfima. El número de publicaciones ha ido en aumento y se pronostica una tendencia general positiva hasta el 2020. Estos resultados sugieren un interés creciente, aunque no consistente en todas las ramas, en la temática de la discapacidad por parte de los graduandos.

En cuanto a las características del estudiantado con discapacidad, es positivo que no haya disparidad considerable por género o provincia de origen y que en su mayoría hayan recibido ayuda financiera por parte de la institución. Tampoco hay diferencias considerables por tipo de discapacidad considerando la distribución nacional, excepto por la infrarrepresentación de las personas con discapacidad intelectual. Pero sí existe una diferencia evidente según el grado de discapacidad. El número de estudiantes con discapacidad aminora a medida que el grado de discapacidad aumenta, lo cual anima a buscar vías para captar y atender estudiantes con discapacidades severas.

Las facultades de Jurisprudencia, Ciencias Médicas y Especialidades Empresariales han atendido a más de la mitad del estudiantado con discapacidad del periodo señalado, mientras que las facultades de carreras técnicas e ingenierías presentan algunos de los porcentajes más bajos. Esto podría deberse a que ciertas facultades o carreras están mejor adaptadas para atender a los estudiantes con discapacidad que otras. También podría señalar la existencia de barreras discriminatorias en ciertas ciencias o incluso de prejuicios propios de los estudiantes con discapacidad. Aunque el número de estudiantes matriculados con discapacidad ha ido en aumento desde el 2013; sin embargo, no ha alcanzado el $1 \%$ de la población estudiantil general hasta el año 2017, dando cuenta del largo camino que queda por recorrer.

Entre las limitaciones de la investigación se reconoce la limitada información existente, lo cual impidió el estudio de la tasa de retención, deserción y egreso de los estudiantes con discapacidad. Se recomienda que en trabajos futuros se investigue la prevalencia de personas con discapacidad en las universidades ecuatoriana a través de los años para que, de esta forma, se pueda evaluar el progreso en inclusión. También se recomienda estudiar los porcentajes de retención, deserción y egreso de este colectivo en la formación terciaria. Otra línea de investigación sugerida son los obstáculos que pueden impedir o dificultar el 
ingreso a las universidades o a un tipo de carreras determinadas. Por último, se exhorta a investigadores, docentes e instituciones por igual a emprender sus propias iniciativas de inclusión educativa, en pos de una sociedad más íntegra, solidaria y justa.

\section{Referencias}

American Psychology Association. (2009). Barriers to students with disabilities in psychology training. Washington DC: Asociación Americana de Psicología.

Aquino, S., García, V. e Izquierdo, J. (2012). La inclusión educativa de ciegos y baja visión en el nivel superior: Un estudio de caso. Sinéctica, 39, 1-21.

Asamblea Nacional de la República del Ecuador. (2010). Ley orgánica de educación superior. Recuperado de https://www.educacionsuperior.gob.ec/

Asamblea Nacional de la República del Ecuador. (2011). Ley orgánica de educación intercultural. Recuperado de https://oig.cepal.org/

Asamblea Nacional de la República del Ecuador. (2012). Ley orgánica de discapacidades. Recuperado de https://oig.cepal.org/

Baquero, M. (2015). Discapacidad: Una construcción narrativa excluyente. Equidad \& Desarrollo, 24, 165-183. https://doi.org/10.19052/ed.3685

Bell, F. (2017). El desarrollo de los procesos sustantivos de la educación superior ecuatoriana ante el reto de la inclusión educativa. Revista Latinoamericana de Educación Inclusiva, 1 1(1), 199212. https://doi.org/10.4067/S07 18-73782017000100012

Bodero, L. y Terranova, E. (2012). La inclusión de los estudiantes con discapacidades en la Universidad Laica Vicente Rocafuerte de Guayaquil. Yachana, 1(1), 103-107.

Booth, T. (2003). Overcoming exclusion through inclusive approaches in education: A challenge and a vision. París: UNESCO.

Castignani, M., Hanlon, P., Luján, M., Katz, S. y Peiró, M. (2014). Comisión universitaria sobre discapacidad: Una experiencia de gestión en la Universidad Nacional de La Plata. Revista Latinoamericana de Educación Inclusiva, 8(1), 51-61.

Comisión Económica para América Latina y el Caribe. (2014). Informe regional sobre la medición de la discapacidad: Una mirada a los procedimientos de medición de la discapacidad en América Latina y el Caribe. Santiago de Chile: Comisión Económica para América Latina y el Caribe.

Consejo Nacional para la Igualdad de Discapacidades. (2017). Análisis general: Personas con discapacidad registradas. Recuperado de http://www.consejodiscapacidades.gob.ec/estadistica/index.html

Crosso, C. (2010). El Derecho a la educación de personas con discapacidad: Impulsando el concepto de educación inclusiva. Revista Latinoamericana de Educación Inclusiva, 4(2), 79-95.

Cruz, R. y Casillas, M. (2017). Las instituciones de educación superior y los estudiantes con discapacidad en México. Revista de Educación Superior, 46(181), 37-53. https://doi.org/10.1016/j.resu.2016.11.002

Cueva, M., Gallardo, J., Romero, A., Falconi, A. y Castro, S. (2017). Consideraciones de personas discapacitadas sobre el acceso a la educación superior en la ciudad de Latacunga, Ecuador. Revista Órbita Pedagógica, 4(2), 49-61.

Espinosa, B. y Goetschel, A. (2014). Construcciones de calidad e igualdad en las universidades ecuatorianas. Nuevos desafíos para la inclusión social y la equidad en la educación superior. 
En M. Rifá, L. Duarte y M. Ponferrada (Ed.), Actas del III congreso internacional MISEAL (págs. 132-145). Barcelona: MISEAL. https://doi.org/10.22206/cys.2012.v37i3.pp255-73

Espinosa, C., Gómez, V. y Cañedo, C. (2012). ¿Integración o inclusión? La educación superior ecuatoriana y el pleno acceso de estudiantes con discapacidad. Ciencia y Sociedad, 37(3), 255273.

Esteves, A. y Santos, D. (2014). Una mirada a la inclusión social de las personas con discapacidad: Experiencia de FLACSO-Ecuador. Nuevos. En M. Rifá, L. Duarte y M. Ponferrada (Ed.), Actas del III congreso internacional MISEAL (págs. 233-251). Barcelona: MISEAL.

Fajardo, S. (2017). La educación superior inclusiva en algunos países de latinoamérica: Avances, obstáculos y retos. Revista Latinoamericana de Educación Inclusiva, 11(1), 171-197. https://doi.org/10.4067/S0718-73782017000100011

Gallegos, M. (2011). La inclusión educativa en la Universidad Politécnica Salesiana. Alteridad, Revista de Educación, 6(2), 118-126.

Hernández-Sampieri, R., Fernández, C. y Baptista, P. (2010). Medología de la investigación. Ciudad de México: McGraw-Hill.

Herrera, J., Parrilla, A., Blanco, A. y Guevara, G. (2018). La formación de docentes para la educación inclusiva. Un reto desde la Universidad Nacional de Educación en Ecuador. Revista Latinoamericana de Educación Inclusiva, 12(1), 21-38. https://doi.org/10.4067/So718-73782018000100021

Instituto Nacional de Estadística e Informática. (2015). Perú: Características de la población con discapacidad. Lima: Instituto Nacional de Estadística e Informática.

Instituto Nacional de Estadística y Censos-INDEC. (2014). Censo nacional de población, hogares y viviendas 2010. censo del bicentenario. Serie C. Buenos Aires: Instituto Nacional de Estadística y Censos.

Instituto Nacional de Estadística y Censos-INEC. (2014). Evolución de las variables investigadas en los censos de población y vivienda del Ecuador 1950, 1962, 1974, 1982, 1990, 2001 y 2010. Quito: Instituto Nacional de Estadística y Censos.

Jiménez, A. y Huete, A. (2016). Informe olivenza 2016, sobre la situación de la discapacidad en España. Madrid: Observatorio Estatal de la Discapacidad.

Kahn, S. y Ginther, D. (2017). Women and STEM. Boston, MA: National Bureau of Economic Research. https://doi.org/10.3386/w23525

Kraus, L. (2017). Disability statistics annual report. Durham, NH: University of New Hampshire.

Lissi, R., Zuzulich, S., Salinas, M., Achiardi, C., Hojas, A. y Pedrals, N. (2009). Discapacidad en contextos universitarios: Experiencia del PIANE UC en la Pontificia Universidad Católica de Chile. Calidad en la Educación, 30, 305-324.

Martínez, C., Silva, R. y Naranjo, B. (2016). Accesibilidad a la información y la inclusión social de personas con discapacidad visual. En M. Espinoza, J. Feyen y E. Timbe (Ed.), Actas del IV congreso ecuatoriano de tecnologías de la información y comunicación (pp. 79-86). Cuenca: MASKANA.

Ministerio de Educación. (2013). Acuerdo núm. 0295-13. Quito: Ministerio de Educación.

Ministerio de Educación. (2017). El 78\% de niños y jóvenes con discapacidad en edad escolar asiste al sistema educativo. Recuperado de https://educacion.gob.ec/el-78-de-ninos-y-jovenes-condiscapacidad-en-edad-escolar-asiste-al-sistema-educativo/ 
Ministerio de Salud Pública. (2015). Registro nacional de discapacidades. Quito: Ministerio de Salud Pública.

Misión Solidaria Manuela Espejo. (2012). Ier Estudio biopsicosocial clínico genético de las personas con discapacidad en Ecuador 2009-2010. Quito: Vicepresidencia de la República del Ecuador.

Moon, N., Todd, R., Morton, D. y Ivey, E. (2012). Accommodating students with disabilities in science, technology, engineering, and mathematics (STEM): Findings from research and practice for middle grades through university education. Atlanta, GA: Center for Assistive Technology and Environmental.

Naciones Unidas. (2006). Conveción internacional sobre los derechos de las personas con discapacidad. París: Naciones Unidas.

National Center for Education Statistics. (2016). Digest of education statistics 2015. Recuperado de https://nces.ed.gov/pubs2016/2016014.pdf

Observatorio Nacional de Discapacidad. (2014). Línea base observatorio nacional de discapacidad: Análisis descriptivo de indicadores. Bogotá: Observatorio Nacional de Discapacidad.

OMS. (2001). Clasificación internacional del funcionamiento, de la discapacidad y de la salud. Ginebra: Organización Mundial de la Salud.

OMS. (2011). Informe mundial sobre la discapacidad 2011. Malta: Organización Mundial de la Salud.

Palacios, A. (2008). El modelo social de discapacidad: Orígenes, caracterización y plasmación en la Convención internacional sobre los derechos de las personas con discapacidad. Madrid: CINCA.

Payà, A. (2010). Políticas de educación inclusiva en América Latina. Propuestas, realidades y retos de futuro. Revista Educación Inclusiva, 3(2), 125-142.

Peralta, A. (2007). Libro blanco sobre universidad y discapacidad. Madrid: Centro Español de Documentación sobre Discapacidad.

Perez, L., Gulley, A. y Prickett, L. (2016). Improving access to higher education with UDL and switch access technology: A case study. En M. Mills y D. Wake (Eds.), Empowering learners with mobile open-access learning initiatives (pp. 13-30). Hershey, PE: IGI Global.

Redacción Sociedad. (3 de Octubre de 2015). Más de 5 mil personas con discapacidad asisten a la universidad en Ecuador. Diario El Telégrafo, p. 26.

Rodríguez, N. (2004). Integración de las personas con discapacidad en la educación superior en Ecuador. Quito: Digital Observatory for Higher Education in Latin America and the Caribbean.

Samaniego, P. (2009). Personas con discapacidad y acceso a servicios educativos en Latinoamérica. Madrid: CINCA.

Secretaría Nacional de Planificación y Desarrollo. (2017). Plan nacional para el buen vivir 2017-2021. Quito: Secretaría Nacional de Planificación y Desarrollo.

Secretaria Nacional de Promoção dos Direitos da Pessoa com Deficiência. (2012). Cartilha do censo 2010. Pessoas com deficiência. Brasilia: Secretaria de Direitos Humanos da Presidência da República.

Servicio Nacional de la Discapacidad. (2016). II Estudio nacional de la discapacidad en Chile. Santiago: Servicio Nacional de la Discapacidad.

Smith, D. (2016). Disability in the United Kingdom 2016: Facts and figures. Cambridge: Papworth Trust.

Stang, M. (2011). Las personas con discapacidad en América Latina. Del reconocimiento jurídico a la desigualdad real. Santiago: Naciones Unidas. 
Universidad Católica de Santiago de Guayaquil. (2017). Planificación estratégica de desarollo institucional 2017-2021. Guayaquil: Universidad Católica de Santiago de Guayaquil.

Vizñay, J., Campoverde, M. y Poma, D. (2017). Funciones de accesibilidad que logran y mejoran las competencias de uso y manejo de los teléfonos inteligentes. En V. Robles, R. Sánchez, P. Ingavélez y F. Pesántez (Coord.), Inclusión, discapacidad y educación: Enfoque práctico desde las tecnologías emergentes (pp. 97-110). Quito: Abya-Yala.

\section{Breve CV del autor}

\section{Juan Carlos Ocampo}

Estudiante investigador de la carrera de Psicología Clínica de la Universidad Católica de Santiago de Guayaquil. Miembro fundador y exdirigente del grupo de investigación científica estudiantil de la Facultad de Filosofía, Letras y Ciencias de la Educación de la universidad mencionada. Ha realizado trabajos de investigación científica en orientación vocacional, violencia de género, sexualidad y discapacidad. También ha colaborado con varias organizaciones, entre ellas el Instituto de Neurociencias de la Junta de Beneficencia de Guayaquil, en temas de investigación y desarrollo de soluciones basadas en evidencia. Actualmente es becario por el Instituto Tecnológico y de Estudios Superiores de Monterrey en la carrera de Psicología Clínica y de la Salud. ORCID ID: https://orcid.org/o000-0002-9353-7581. Email: juan.ocampo01@cu.ucsg.edu.ec 